Check for updates

Cite this: RSC Adv., 2017, 7, 48120

Received 31st July 2017

Accepted 23rd September 2017

DOI: $10.1039 / c 7 r a 08435 h$

rsc.li/rsc-advances

\section{Viscoelastic properties of supramolecular gemini- like surfactant solutions in the absence of inorganic salts $\dagger$}

\begin{abstract}
Danhua Xie, (D) *a Donghong You, ${ }^{\text {* }}$ Shaoming Ying, ${ }^{a}$ Binglei Song ${ }^{b}$ and Jinnian Tian $^{\mathrm{b}}$
Exploration of novel wormlike micelle systems is important for both the theory and applications of surfactant self-assembly. A new anionic surfactant, sodium 2-phenoxyhexadecanoate (sodium Ph-C16), was synthesized in this work. This surfactant can self-assemble in solution to form wormlike micelles with the help of a bolaform salt, ethane-1,2-bis(trimethyl ammonium bromide) ( $\mathrm{N}-2-\mathrm{N}$ bromide), by removing inorganic salts. The resultant aqueous system was investigated using rheological measurements, freeze-fractured-TEM and 2D NOESY ${ }^{1} \mathrm{H}$ NMR experiments. A supramolecular geminilike structure constructed from electrostatic interactions was a prerequisite for the wormlike micelle formation. The zero shear viscosity $\left(\eta_{0}\right)$ of the system had a very strong dependence on the surfactant concentration with a scaling exponent of 16.8 in the semi-dilute region, which is attributed to the extremely strong electrostatic attraction between the surface-active anions and bolaform cations. These results are beneficial for understanding anionic wormlike micelles in the absence of inorganic salts.
\end{abstract}

\section{Introduction}

Wormlike micelles are a type of supramolecular aggregate formed by surfactants under suitable conditions. ${ }^{\mathbf{1}}$ These aggregates can have dimensions that are hundreds or even thousands of nanometers in size, which imparts remarkable viscoelasticity to aqueous solutions. As living polymers, wormlike micelles have wide applications as fracturing fluids in oil fields, drag reducing agents and shampoos. The most commonly reported wormlike micelles are those that form in cationic surfactant aqueous solutions in the presence of salts ${ }^{2-8}$ and in cationic-anionic ${ }^{9-11}$ surfactant systems. In these systems, a suitable molecular geometry can be obtained by screening the head group charge of the surfactant counter ions, which is beneficial for wormlike micelle formation. ${ }^{\mathbf{1 2 - 1 6}}$

In recent years, investigations on inorganic salt-free surfactant systems in aqueous solutions have generated useful results on novel aggregate morphology and phase behavior. ${ }^{17-21}$ Because of the absence of inorganic salts, the ionic strength in the systems is quite low, resulting in unscreened aggregates and high osmotic pressures. ${ }^{22}$ Zemb et al. discovered aggregates of

${ }^{a}$ Fujian Provincial Key Laboratory of Featured Materials in Biochemical Industry, College of Chemistry and Materials, Ningde Normal University, Ningde, Fujian, 352100, P. R. China. E-mail: danhuaxie@ndnu.edu.cn

${ }^{b}$ Key Laboratory of Synthetic and Biological Colloids, Ministry of Education, School of Chemical \& Materials Engineering, Jiangnan University, Wuxi, Jiangsu, 214122, P. R. China

$\dagger$ Electronic supplementary information (ESI) available: Synthesis details of sodium Ph-C16 and N-2-N bromide and showing the steady shear viscosity curve of sodium Ph-C16 at $200 \mathrm{mmol} \mathrm{L}^{-1}$. See DOI: 10.1039/c7ra08435h nanodisks and hollow icosahedra in catanionic salt-free systems. ${ }^{23-25}$ Hao et al. reported an onion phase in such systems. ${ }^{26}$ It is obvious that salt-free systems represent systems where electrostatic interactions occur under extreme conditions. Investigating stable wormlike micelles in inorganic saltfree systems can help further understanding of the effect of charge on wormlike micelle growth and evolution. However, the most common morphologies in inorganic salt-free systems are vesicles and liquid crystal phases. Wormlike micelles are only occasionally reported in these systems and usually only exist in a very narrow mixture ratio or concentration range. ${ }^{17,19,27-29}$

Gemini surfactants, which are composed of two alkyl chains, two head groups and a spacer, have an advantageous molecular geometry and can form wormlike micelles without the addition of salts. ${ }^{15,16,30}$ To avoid a tedious synthesis of new, structured gemini surfactants, a strategy has been developed to construct wormlike micelles using pseudo-gemini surfactants ${ }^{\mathbf{1 9 , 2 1 , 3 1 - 3 7}}$ with gemini-like structures that are formed via intermolecular electrostatic interactions. Inspired by this strategy, a new anionic surfactant, sodium 2-phenoxyhexadecanoate (sodium Ph-C16), was synthesized in this work (Fig. 1) and used to construct supramolecular gemini surfactant structures with a bolaform electrolyte, ethane-1,2-bis(trimethyl ammonium bromide), abbreviated $\mathrm{N}-2-\mathrm{N}$ bromide. In contrast to previously reported systems, ${ }^{19,21,31-36}$ the inorganic salts were removed in the present system. Wormlike micelle formation was observed over a wide range of concentrations in this inorganic salt-free system (di-Ph-C16/N-2-N), which provided insight into the properties of wormlike micelles in the absence of inorganic salts. 


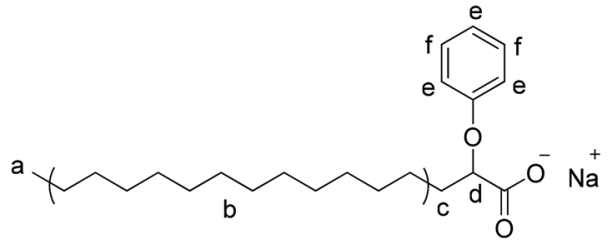

(a)<smiles>C[N+](C)(C)CC[N+](C)(C)Br</smiles>

(b)

Fig. 1 The molecular structures of (a) sodium 2-phenoxyhexadecanoate, abbreviated sodium Ph-C16 and (b) ethane-1,2-bis(trimethyl ammonium bromide), abbreviated N-2-N bromide.

\section{Materials and methods}

\subsection{Materials}

All compounds used, including the palmitic acid, $\mathrm{SOCl}_{2}, \mathrm{Br}_{2}$, and phenol, were purchased from Aladdin Reagent Co., Ltd. The reagents and solvents used were analytical reagent grade. Sodium 2-phenoxyhexadecanoate and the bolaform salt, ethane-1,2-bis(trimethyl ammonium bromide), were synthesized in our lab. The detailed synthetic processes and NMR data can be found in the ESI. $\dagger$

\subsection{Sample preparation}

The ethane-1,2-bis(trimethyl ammonium hydroxide) solution (N-2-N hydroxide) was prepared from an ethane-1,2bis(trimethyl ammonium bromide) aqueous solution via ion exchange using a strong basic anion exchanger (Amberlite ${ }^{\circledR}$ IRA 402). To ensure the solution was acceptable for further use, it was tested with a nitric acid-acidified $\mathrm{AgNO}_{3}$ solution to ensure no precipitates appeared. The surfactant, sodium Ph-C16, was acidified with $\mathrm{HCl}$ to obtain its acid form. A certain amount of $\mathrm{N}-2-\mathrm{N}$ hydroxide was neutralized with the Ph-C16 acid. After freeze-drying, the products were used to prepare a di-Ph-C16/N2-N solution.

\subsection{Rheology measurements}

Rheological measurements were performed using a stresscontrolled rheometer (Discovery DHR-2, TA instruments) with a cone-plate sensor. The cone was made of standard ETC steel with a diameter of $40 \mathrm{~mm}$ and a cone angle of $2^{\circ}$. The gap between the center of the cone and the plate was $48 \mu \mathrm{m}$. Each sample was allowed to equilibrate on the plate for $5 \mathrm{~min}$ before testing. A strain sweep was performed at a frequency of $2.82 \mathrm{rad} \mathrm{s}$ before each test. A strain value was determined and used to ensure that the sample was in the linear viscoelastic region during the oscillatory measurements. The temperature was maintained at $25{ }^{\circ} \mathrm{C}$ throughout the experiments. All the solutions were prepared using Milli-Q water with a resistivity of $18.2 \mathrm{M} \Omega \mathrm{cm}$.

\subsection{D NOESY ${ }^{1} \mathrm{H}$ NMR measurements}

2D NOESY measurements was performed on a Bruker Avance III 400 spectrometer operating at $400 \mathrm{MHz}$. Deuterium oxide (99.9\%) was purchased from Cambridge Isotope Laboratories and was used to prepare the di-Ph-C16/N-2-N solution in $\mathrm{D}_{2} \mathrm{O}$. The center of the HDO signal (4.79 ppm) was used as the reference. The experiments were performed with a standard three-pulse sequence and a mixing time of $300 \mathrm{~ms}$.

\subsection{FF-TEM}

The samples were prepared using freeze-fractured methods. A small amount of sample was placed on a $0.1 \mathrm{~mm}$ thick copper disk. The sample was quickly frozen by plunging it into liquid propane cooled by liquid nitrogen. The fracturing and replication were performed on a freeze-fracture apparatus (BalzersBAF400, Germany) at $-160{ }^{\circ} \mathrm{C}$. Carbon was deposited at an angle of $90^{\circ}$, and then, platinum was deposited at an angle of $45^{\circ}$ to shadow the replicas. After removing the organic components, the resulting replicas were observed with a JEM-2100 electron microscope $(200 \mathrm{kV})$.

\section{Results and discussion}

\subsection{Steady state rheological measurements of the di-Ph-C16/ N-2-N system}

Steady shear viscosity measurements are widely used to probe the viscoelastic behavior of surfactant solutions. Fig. 2 shows the steady shear viscosity curves of the di-Ph-C16/N-2-N system at different Ph-C16 ion concentrations. As observed in Fig. 2, the viscosity was constant at low concentrations $\left(C \leq 40 \mathrm{mmol} \mathrm{L}^{-1}\right)$ as the shear rate increased, and the solution exhibited a Newtonian behavior. At higher concentrations, the viscosity of the di-Ph-C16/N-2-N system decreased with the increasing shear rate. The shear-thinning behavior indicates the presence of wormlike micelles. ${ }^{38}$ The viscosity value that corresponds to the plateau in the steady shear viscosity curve at low shear rates is called the zero shear viscosity $\left(\eta_{0}\right)$. Over the range of the examined concentrations, the largest $\eta_{0}$ was $16.2 \mathrm{~Pa} \mathrm{~s}$ at

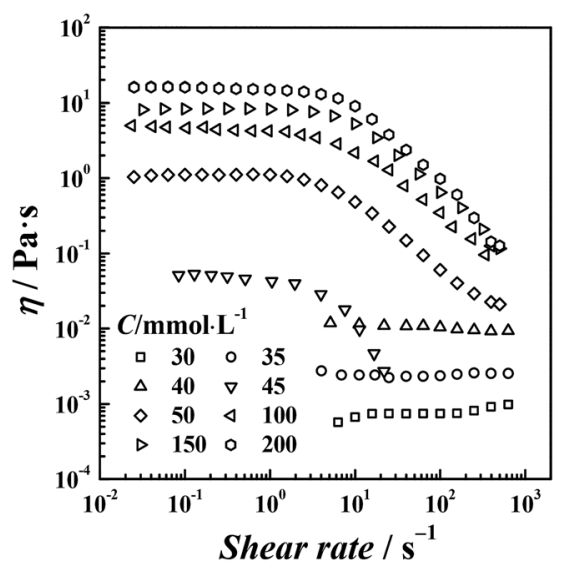

Fig. 2 Steady shear viscosity curves of di-Ph-C16/N-2-N at different $\mathrm{Ph}-\mathrm{C} 16$ ion concentrations. 
$200 \mathrm{mmol} \mathrm{L}^{-1}$. However, the sodium $\mathrm{Ph}-\mathrm{C} 16$ solution remains liquid-like with an $\eta_{0}$ value of $1.5 \mathrm{mPa} s$ at the same concentration (ESI Fig. S2 $\dagger$ ). A similar system using the traditional hexadecanoate ion instead of Ph-C16 ion also gives an $\eta_{0}$ value of $1.5 \mathrm{mPa} \mathrm{s}$ (ESI Fig. S3†). These comparisons reveal the peculiarity of di-Ph-C16/N-2-N system. Firstly, the di-cations with a short spacer cause the strong viscoelasticity in the diPh-C16/N-2-N system. Two anionic surfactant ions are drawn close by one $\mathrm{N}-2-\mathrm{N}$ molecule via electrostatic attractions. A supramolecular gemini-like structure formed and led to the formation of wormlike micelles. ${ }^{39}$ Secondly, the phenyl group contained in the Ph-C16 ion enhances the molecular interactions and finely tuned the molecular geometry, also facilitating the wormlike micelle formation.

\subsection{The scaling behavior of the di-Ph-C16/N-2-N system}

In the wormlike micelle formation process, the spherical micelles that initially form gradually transform into rod-like micelles as the concentration increases. When the amount and length of the elongated aggregates reach a critical state, entanglements occur and impart the solution with viscoelasticity. The concentration at which the zero shear viscosity $\left(\eta_{0}\right)$ begins to show an obvious increase is called the overlap concentration. ${ }^{40}$ As shown in Fig. 3, that concentration is $35 \mathrm{mmol} \mathrm{L}^{-1}$ in this system. Then, the systems enter the semidilute regime, which is where $\eta_{0}$ rapidly increases with the concentration. The dependence of $\eta_{0}$ on the concentration, $C$, can be described by a power law: $\eta_{0} \propto C^{\alpha}$, where $\alpha$ is the exponent. $^{\mathbf{4 1 , 4 2}}$ The di-Ph-C16/N-2-N system has an exponent of 16.8 , indicating the extremely strong concentration dependence of $\eta_{0}$. This result is understandable because the di-Ph-C16/N-2$\mathrm{N}$ system is an inorganic salt-free system ${ }^{43-46}$ with a low ionic strength. The surface charge of the wormlike micelles is large compared with that of the micelles in conventional surfactant systems. The electrostatic interactions contribute to the free energy of the end caps, which accelerates the growth of the wormlike micelles. ${ }^{47}$ When the Ph-C16 ion concentration reaches $50 \mathrm{mmol} \mathrm{L}^{-1}$ in the di-Ph-C16/N-2-N system, the variations in $\eta_{0}$ as the concentration changes gradually level off, indicating

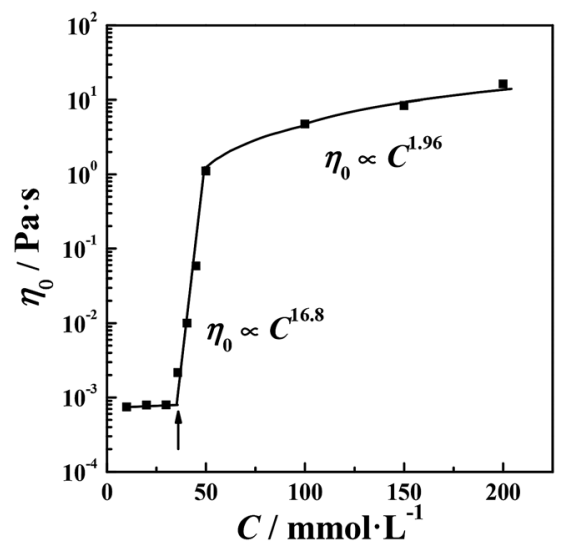

Fig. 3 The variation in the zero shear viscosity $\left(\eta_{0}\right)$ as a function of the concentration. the microstructure transition in the solution. This phenomenon is different from that in most wormlike micelle systems, where $\eta_{0}$ goes through a maximum and then promptly decreases with the concentration because of micelle branching. ${ }^{48}$ The weak dependence of $\eta_{0}$ on the concentration indicates that the average wormlike micelle length no longer obviously increases with the concentration. In addition, the scaling law gives an exponent of 1.96. This value is comparable with the value of 1.5 predicted using the polyelectrolyte solution theory ${ }^{49}$ and reflects the effect of the charge carried by the wormlike micelles on the rheological behavior of the solution. When wormlike micelles entangle, the repulsions between the charged segments in the wormlike micelles makes the fusion of the micelles with each other difficult. The production of joints, which are a key constituent of branched micelles, is more difficult than that in neutral wormlike micelles. The relaxation process of joints sliding along the contour of elongated micelles is also suppressed. Although micelle branching is still inevitable, a multi-connected network is a more suitable description of the microstructure in this region, and this has also been investigated in several systems. ${ }^{\mathbf{1 6 , 5 0}}$ In the present system, this phenomenon is a direct result of the absence of inorganic salts.

\subsection{The dynamic rheological behavior of the di-Ph-C16/N-2- N system}

Wormlike micelles participate in dynamic scission and recombination processes. The slowly increasing region of $\eta_{0}$ in the diPh-C16/N-2-N system was further investigated via oscillatory shear measurements to obtain further information about the rheological properties. In most cases, the relaxation processes can be characterized by the Maxwell model with a single relaxation time, ${ }^{\mathbf{4 1 , 5 1}}$ which is presented by the following equations:

$$
\begin{aligned}
G^{\prime} & =\frac{\left(\omega \tau_{\mathrm{R}}\right)^{2}}{1+\left(\omega \tau_{\mathrm{R}}\right)^{2}} G_{0} \\
G^{\prime \prime} & =\frac{\omega \tau_{\mathrm{R}}}{1+\left(\omega \tau_{\mathrm{R}}\right)^{2}} G_{0}
\end{aligned}
$$

where $G^{\prime}$ is the elastic modulus, $G^{\prime \prime}$ is the viscous modulus, $G_{0}$ is the plateau modulus, $\tau_{\mathrm{R}}$ is the characteristic relaxation time, and $\omega$ is the oscillation angular frequency. As shown in Fig. 4(a), the elastic modulus $\left(G^{\prime}\right)$ is above the viscous modulus $\left(G^{\prime \prime}\right)$ at high frequencies and below $G^{\prime \prime}$ at low frequencies. According to the Maxwell model, the frequency where two moduli cross each other is denoted $\omega_{\mathrm{c}}$, and the reciprocal of this value can be used to estimate the relaxation time, $\tau_{\mathrm{R}}$. Fig. $4(\mathrm{~b})$ shows the variation of $G^{\prime \prime}$ with $G^{\prime}$ (Cole-Cole plot) in the di-Ph-C16/N-2-N system, and the variation follows a perfect semicircle at low frequencies, indicating that the system behaves like a Maxwell fluid. The data deviation at higher frequencies implies the appearance of other relaxation mechanisms, such as Rouse-like motion.

The relaxation time, $\tau_{\mathrm{R}}$, characterizes the timescale of a wormlike micelle experiencing relaxation processes and can be related to the average micelle length. ${ }^{52}$ As shown in Fig. 5, the $\tau_{\mathrm{R}}$ slightly decreases with the increasing concentration, and this behavior is caused by the partial branching of the wormlike 

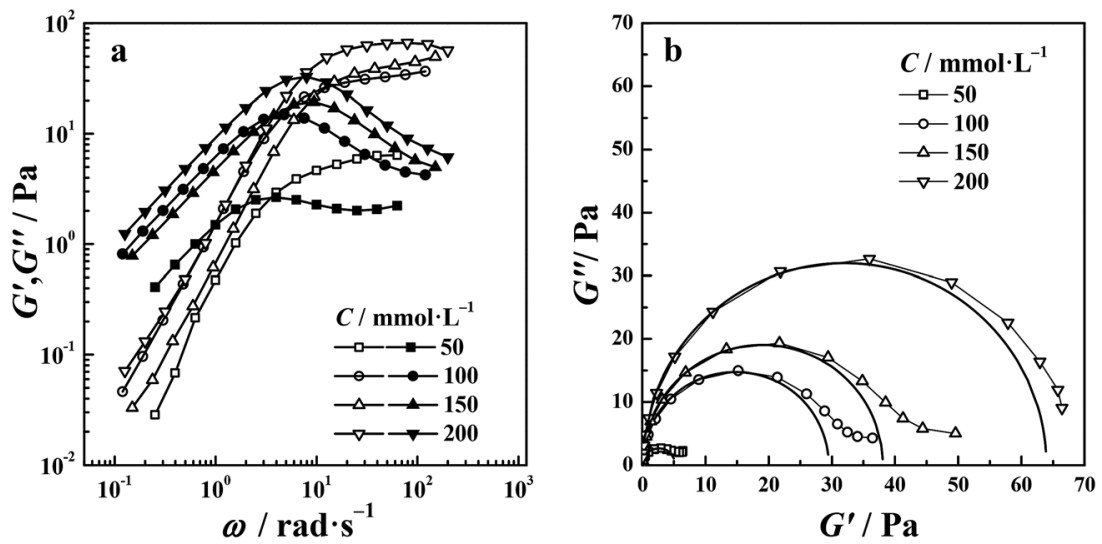

Fig. 4 (a) Dynamic rheological spectra and (b) the Cole-Cole plots of the di-Ph-C16/N-2-N system (filled and open symbols in (a) represent the elastic modulus $\left(G^{\prime}\right)$ and viscous modulus $\left(G^{\prime \prime}\right)$, respectively).

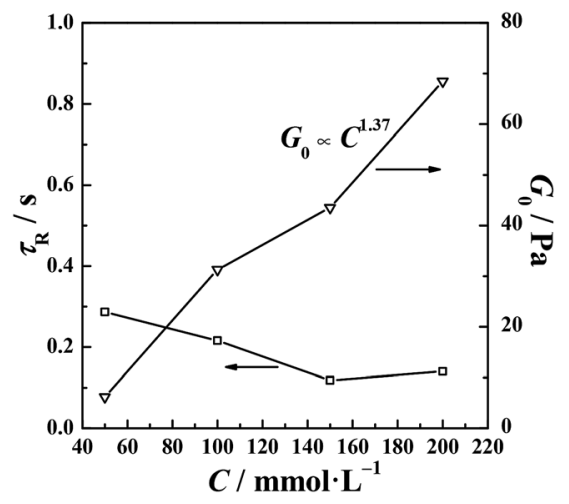

Fig. 5 The relaxation time, $\tau_{\mathrm{R}}$, and plateau modulus, $\mathrm{G}_{0}$, of the di-Ph$\mathrm{C} 16 / \mathrm{N}-2-\mathrm{N}$ systems as a function of the $\mathrm{Ph}-\mathrm{C} 16$ ion concentration.

micelles, which was discussed above. The joints that are produced can slide along the micelle body and serve as a stress relaxation pathway. The diffusion of the wormlike micelles becomes faster, mimicking the behavior of micelles with shorter lengths. The elasticity of the aqueous solutions comes from the entanglement of the wormlike micelles. The plateau modulus, $G_{0}$, is used to evaluate the number density of the entanglement points. The slow increase in $G_{0}$ indicates the entanglements are enhanced with the increasing concentration. The above phenomenon can also been perceived as the switching in relaxation mode. ${ }^{53}$ The scaling law calculated an exponent of 1.37 , which is close to the value of 1.5 predicted for polyelectrolyte solutions in the region of chain entanglement. ${ }^{49,54}$ This similarity in the scaling behavior reveals the charged nature of the investigated wormlike micelles.

\subsection{FF-TEM images}

The wormlike micelles formed in the di-Ph-C16/N-2-N system were further investigated using FF-TEM (Fig. 6). Massive threadlike micelles with a cross-sectional diameter of approximately $3 \mathrm{~nm}$ are densely entangled, preventing an estimation of the average micelle length of the wormlike micelles. Some connection joints can also be observed. The concentration of
$50 \mathrm{mmol} \mathrm{L}{ }^{-1}$ characterizes the transition from the quickly increasing region to the slowly increasing region of $\eta_{0}$. The direct morphology image at this concentration showed that the wormlike micelles were entangled below this concentration.

\subsection{D NOESY experiments on the di-Ph-C16/N-2-N system}

The formation of wormlike micelles by $\mathrm{Ph}-\mathrm{C} 16$ ions requires the electrostatic attractions of counter di-cations. The interactions between groups are different in the presence or absence of $\mathrm{N}-2$ $\mathrm{N}$ ions. The 2D NOESY experiments were performed to obtain a clear image of the supramolecular gemini-like surfactant constructed via electrostatic interactions. The cross-peak signals of protons in the $2 \mathrm{D}$ spectrum indicate the distance between protons within $5 \AA \AA^{55,56}$ providing direct evidence of molecular interactions. The 2D NOESY spectra of the sodium

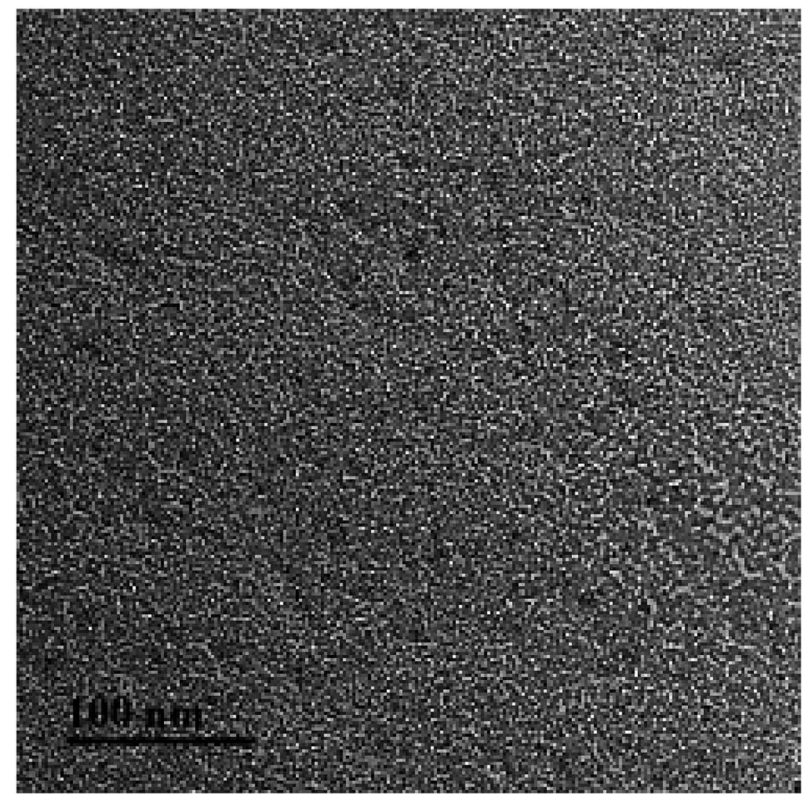

Fig. 6 The FF-TEM image of the di-Ph-C16/N-2-N system (concentration of the $\mathrm{Ph}-\mathrm{C} 16$ ion: $50 \mathrm{mmol} \mathrm{L}^{-1}$ ). 
Ph-C16 and di-Ph-C16/N-2-N system at the same Ph-C16 ion concentration $\left(5 \mathrm{mmol} \mathrm{L}^{-1}\right)$ are shown in Fig. 7 . In the absence of $\mathrm{N}-2-\mathrm{N}$ (Fig. 7(a)), the cross-peak signals are between the adjacent protons of the alkyl chains (b-H, c-H, and $\mathrm{d}-\mathrm{H}$, marked with a square). Because both the phenyl ring and the alkyl chain are hydrophobic in nature, the phenyl ring tends to bend toward the alkyl chain through the $\mathrm{C}-\mathrm{O}-\mathrm{C}$ bond. As a result, a weak signal from the interaction of $\mathrm{d}-\mathrm{H}$ and $\mathrm{e}-\mathrm{H}$ is observed (marked with a rhombus). While in the di-Ph-C16/N-2-N system (Fig. 7(b)), a new set of cross-peak signals arise, indicating the appearance of strong interactions between the phenyl ring and the alkyl chain (marked with an ellipse). This interaction obviously attributed to the effect of the $\mathrm{N}-2-\mathrm{N}$ ions because these signals were not observed in the sodium Ph-C16 solution. A reasonable explanation for this observation is that one $\mathrm{N}-2-\mathrm{N}$ ion bridges two Ph-C16 ions, drawing them close together and enhancing their hydrophobic interactions. The phenyl ring in one molecule has more opportunities to interact with the alkyl chain in the other molecule, resulting in the cross-peak signals of the protons in the 2D NOESY spectrum. The experiment also reveals that although the phenyl ring is located near the ionic head group, it resides in the hydrophobic region of the aggregate and serves as a hydrophobic driving force for wormlike micelle formation.

\subsection{The effect of inorganic salt on the behavior of the di-Ph- C16/N-2-N system}

Salt additions usually enhance the viscoelasticity of wormlike micellar solutions. However, when equal molar $\mathrm{NaBr}$

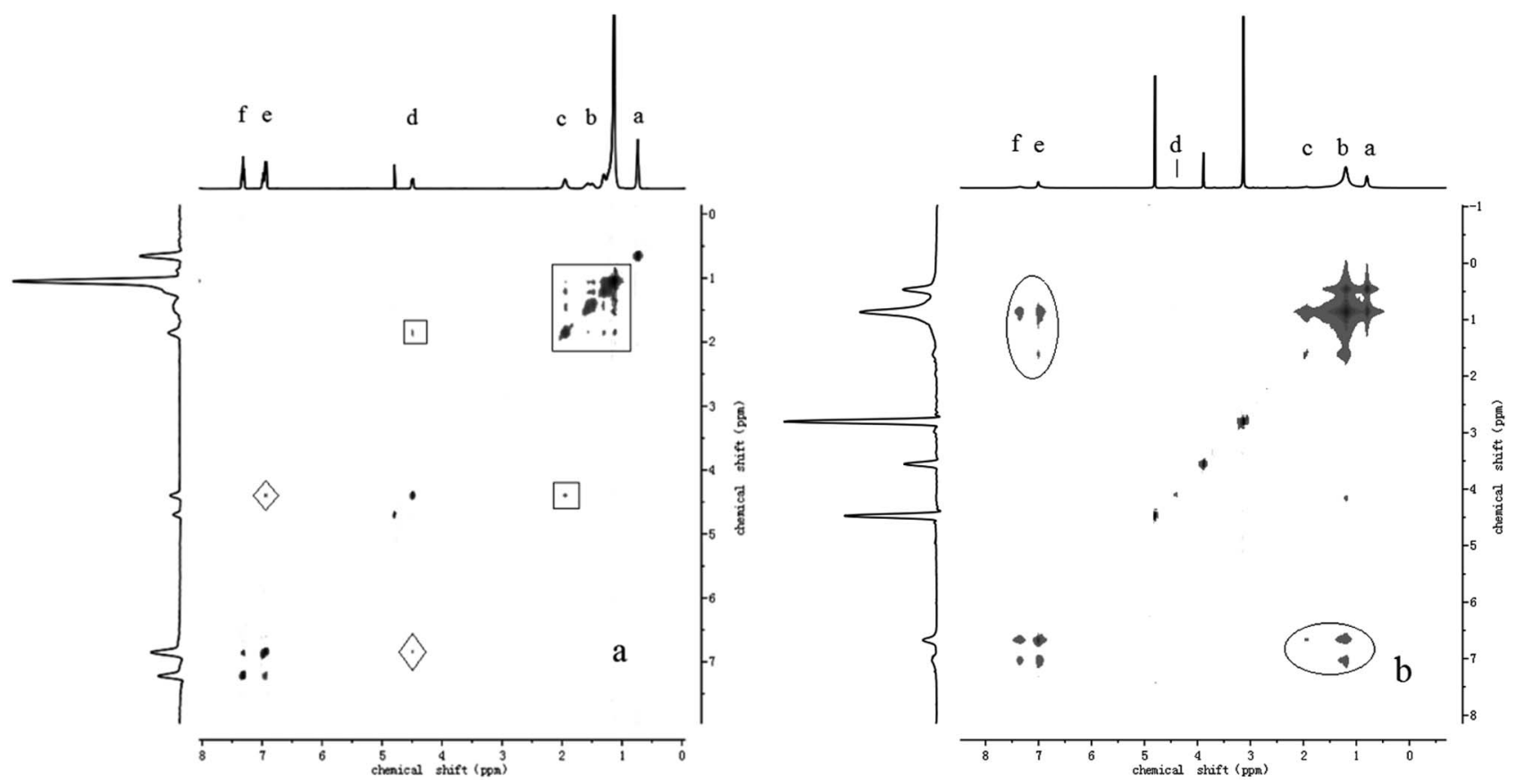

Fig. 7 The 2D NOESY spectra of the (a) sodium Ph-C16 and (b) di-Ph-C16/N-2-N systems (concentration of Ph-C16 ion: 5 mmol L ${ }^{-1}$ ).


Fig. 8 (a) Steady shear viscosity curves of di-Ph-C16/N-2-N with equal molar $\mathrm{NaBr}$; (b) the variation in $\eta_{0}$ with the concentration of di-Ph-C16/ $\mathrm{N}-2-\mathrm{N}$ with equal molar $\mathrm{NaBr}$. 
(NaBr : Ph-C16 ion = $1: 1$ ) was added into the di-Ph-C16/N-2-N system, the zero shear viscosity decreased at all investigated concentrations, as shown in Fig. 8(a). The scaling exponent was also far smaller than that of an inorganic salt-free system (Fig. 8(b)). This comparison shows the advantage of a system without an inorganic salt and reflects the importance of dications in promoting the formation of wormlike micelles. Although the addition of $\mathrm{NaBr}$ is beneficial for the growth of wormlike micelles via screening the ionic head group, the competitive binding of sodium ions with the Ph-C16 ions weakens the attractions between the $\mathrm{N}-2-\mathrm{N}$ and Ph-C16 ions. The supramolecular, gemini-like Ph-C16 ion pairs, which are held together through the electrostatic attractions of $\mathrm{N}-2-\mathrm{N}$, are partially destroyed or weakened. As a result, the viscoelasticity of the system decreased.

\section{Conclusions}

The viscoelastic properties of an inorganic salt-free di-Ph-C16/ $\mathrm{N}-2-\mathrm{N}$ system were investigated. A bola-type cation and a newly synthesized anionic surfactant together generate a supramolecular gemini-like structure via electrostatic interactions, which facilitates the formation of wormlike micelles. Due to the absence of inorganic salts, an extremely high scaling exponent was calculated for the relationship of $\eta_{0}$ and the concentration, which revealed the strong effect of charge on the growth and evolution of the aggregates. The investigated system provides an example of wormlike micelles formed by pseudogemini surfactants in the absence of inorganic salts over a wide concentration range, and these results expand the known conditions for wormlike micelle formation. In addition, the investigated system is an anionic surfactant-dominated wormlike micelle system, which is rarely reported. ${ }^{57-61}$ The exploration of new anionic surfactant systems is beneficial to understand anionic wormlike micelles and to determine and develop their advantages for applications.

\section{Conflicts of interest}

There are no conflicts to declare.

\section{Acknowledgements}

Support from the Foundation of Fujian Educational Committee (JK2015054, JA14325) and the Natural Science Foundation of Fujian Province (grant No. 2014J01052) are gratefully acknowledged.

\section{References}

1 C. A. Dreiss, Soft Matter, 2007, 3, 956-970.

2 W. J. Kim and S. M. Yang, J. Colloid Interface Sci., 2000, 232, 225-234.

3 T. Shikata, H. Hirata and T. Kotaka, Langmuir, 1987, 3, 10811086.

4 T. Shikata, H. Hirata and T. Kotaka, Langmuir, 1988, 4, 354359.
5 T. Shikata, H. Hirata and T. Kotaka, Langmuir, 1989, 5, 398405.

6 F. Kern, P. Lemarechal, S. J. Candau and M. E. Cates, Langmuir, 1992, 8, 437-440.

7 A. Khatory, F. Lequeux, F. Kern and S. J. Candau, Langmuir, 1993, 9, 1456-1464.

8 G. Porte, Y. Poggi, J. Appell and G. Maret, J. Phys. Chem., 1984, 88, 5713-5720.

9 H. Yin, Y. Lin and J. Huang, J. Colloid Interface Sci., 2009, 338, 177-183.

10 H. Fan, Y. Yan, Z. Li, Y. Xu, L. Jiang, L. Xu, B. Zhang and J. Huang, J. Colloid Interface Sci., 2010, 348, 491-497.

11 M. Bergstrom and J. S. Pedersen, Langmuir, 1999, 15, 22502253.

12 V. M. Garamus, Langmuir, 2003, 19, 7214-7218.

13 V. Croce, T. Cosgrove, C. A. Dreiss, G. Maitland, T. Hughes and G. Karlsson, Langmuir, 2004, 20, 7984-7990.

14 D. P. Acharya, H. Kunieda, Y. Shiba and K. Aratani, J. Phys. Chem. B, 2004, 108, 1790-1797.

15 F. Kern, F. Lequeux, R. Zana and S. J. Candau, Langmuir, 1994, 10, 1714-1723.

16 B. Song, Y. Hu, Y. Song and J. Zhao, J. Colloid Interface Sci., 2010, 341, 94-100.

17 H. Li and J. Hao, J. Phys. Chem. B, 2008, 112, 10497-10508.

18 J. C. Hao and H. Hoffmann, Curr. Opin. Colloid Interface Sci., 2004, 9, 279-293.

19 Y. Li, H. Li, J. Chai, M. Chen, Q. Yang and J. Hao, J. Colloid Interface Sci., 2016, 472, 157-166.

20 X. Liu, J. Wang, Z. Cui, H. Yao, X. Ge, W. Chen and F. Sun, RSC Adv., 2017, 7, 26440-26445.

21 H. Lu, Q. Shi, B. Wang and Z. Huang, Colloids Surf., A, 2016, 494, 74-80.

22 H. Li and J. Hao, J. Phys. Chem. B, 2009, 113, 2371-2377.

23 T. Zemb, M. Dubois, B. Deme and T. Gulik-Krzywicki, Science, 1999, 283, 816-819.

24 M. Dubois, B. Deme, T. Gulik-Krzywicki, J. C. Dedieu, C. Vautrin, S. Desert, E. Perez and T. Zemb, Nature, 2001, 411, 672-675.

25 M. Dubois, V. Lizunov, A. Meister, T. Gulik-Krzywicki, J. M. Verbavatz, E. Perez, J. Zimmerberg and T. Zemb, Proc. Natl. Acad. Sci. U. S. A., 2004, 101, 15082-15087.

26 A. X. Song, S. L. Dong, X. F. Jia, J. C. Hao, W. M. Liu and T. B. Liu, Angew. Chem., Int. Ed., 2005, 44, 4018-4021.

27 H. Li, S. A. Wieczorek, X. Xin, T. Kalwarczyk, N. Ziebacz, T. Szymborski, R. Holyst, J. Hao, E. Gorecka and D. Pociecha, Langmuir, 2010, 26, 34-40.

28 P. A. Hassan, B. S. Valaulikar, C. Manohar, F. Kern, L. Bourdieu and S. J. Candau, Langmuir, 1996, 12, 4350-4357.

29 K. Horbaschek, H. Hoffmann and C. Thunig, J. Colloid Interface Sci., 1998, 206, 439-456.

30 Q. Li, X. Wang, X. Yue and X. Chen, Soft Matter, 2013, 9, 9667-9674.

31 Z. Chu and Y. Feng, Chem. Commun., 2010, 46, 9028-9030.

32 J.-X. Zhao and D.-P. Yang, Acta Phys.-Chim. Sin., 2012, 28, 1218-1222.

33 Y. Feng and Z. Chu, Soft Matter, 2015, 11, 4614-4620. 
34 Y. Zhang, P. An, A. Qin, J. Li, X. Lu and X. Liu, Colloid Polym. Sci., 2016, 294, 1743-1754.

35 X. Wang, Z. Zhang, Y. Cao and J. Hao, J. Colloid Interface Sci., 2017, 491, 64-71.

36 J. Wang, Z. Huang, C. Zheng and H. Lu, J. Dispersion Sci. Technol., 2017, 1-6, DOI: 10.1080/01932691.2016.1277431.

37 L. Zhu, Y. Tang and Y. Wang, J. Surfactants Deterg., 2016, 19, 237-247.

38 S. R. Raghavan and E. W. Kaler, Langmuir, 2001, 17, 300-306.

39 J. N. Israelachvili, D. J. Mitchell and B. W. Ninham, J. Chem. Soc., Faraday Trans. 2, 1976, 72, 1525-1568.

40 R. Oda, J. Narayanan, P. A. Hassan, C. Manohar, R. A. Salkar, F. Kern and S. J. Candau, Langmuir, 1998, 14, 4364-4372.

41 M. E. Cates, Macromolecules, 1987, 20, 2289-2296.

42 M. S. Turner and M. E. Cates, J. Phys. II, 1992, 2, 503-519.

43 A. A. Ali and R. Makhloufi, Colloid Polym. Sci., 1999, 277, 270-275.

44 R. G. Shrestha, L. K. Shrestha and K. Aramaki, J. Colloid Interface Sci., 2007, 311, 276-284.

45 E. Cappelaere, R. Cressely and J. P. Decruppe, Colloids Surf., A, 1995, 104, 353-374.

46 S. J. Candau, A. Khatory, F. Lequeux and F. Kern, J. Phys. IV, 1993, 3, 197-209.

47 F. C. MacKintosh, S. A. Safran and P. A. Pincus, Europhys. Lett., 1990, 12, 697.
48 S. A. Rogers, M. A. Calabrese and N. J. Wagner, Curr. Opin. Colloid Interface Sci., 2014, 19, 530-535.

49 M. Rubinstein, R. H. Colby and A. V. Dobrynin, Phys. Rev. Lett., 1994, 73, 2776-2779.

50 A. A. Ali and R. Makhloufi, Phys. Rev. E, 1997, 56, 4474-4478.

51 R. Granek and M. E. Cates, J. Chem. Phys., 1992, 96, 47584767.

52 M. E. Cates, J. Phys., 1988, 49, 1593-1600.

53 K. Morishima, S. Sugawara, T. Yoshimura and M. Shibayama, Langmuir, 2017, 33, 6084-6091.

54 A. V. Dobrynin, R. H. Colby and M. Rubinstein, Macromolecules, 1995, 28, 1859-1871.

55 Y. Fan, Y. Hou, J. Xiang, D. Yu, C. Wu, M. Tian, Y. Han and Y. Wang, Langmuir, 2011, 27, 10570-10579.

56 H. Kessler, M. Gehrke and C. Griesinger, Angew. Chem., Int. Ed., 1988, 27, 490-536.

57 L. J. Magid, Z. Li and P. D. Butler, Langmuir, 2000, 16, 1002810036.

58 P. A. Hassan, S. R. Raghavan and E. W. Kaler, Langmuir, 2002, 18, 2543-2548.

59 G. C. Kalur and S. R. Raghavan, J. Phys. Chem. B, 2005, 109, 8599-8604.

60 K. Nakamura and T. Shikata, Langmuir, 2006, 22, 9853-9859.

61 Y. Han, Y. Feng, H. Sun, Z. Li, Y. Han and H. Wang, J. Phys. Chem. B, 2011, 115, 6893-6902. 